\title{
Teores de Carbono de Cinco Espécies Florestais e seus Compartimentos
}

\author{
Felipe Scheibe Dallagnol ${ }^{1}$, Francelo Mognon ${ }^{1}$, \\ Carlos Roberto Sanquetta ${ }^{2}$, Ana Paula Dalla Corte ${ }^{3}$ \\ ${ }^{1}$ Programa de Pós-graduação em Ciência Florestal, Universidade Federal do Paraná - UFPR \\ ${ }^{2}$ Departamento de Ciências Florestais, Universidade Federal do Paraná - UFPR \\ ${ }^{3}$ Fundação de Pesquisas Florestais do Paraná - FUPEF
}

\begin{abstract}
RESUMO
A quantificação da biomassa e do carbono é importante para a avaliação do potencial de fixação em espécies florestais. Como se sabe, parte da biomassa corresponde ao carbono que pode ser convertido ao equivalente de $\mathrm{CO}_{2}$ para aplicação em projetos de Mecanismo de Desenvolvimento Limpo (MDL) e outros mercados de carbono. Este trabalho teve como objetivo avaliar os teores de carbono de cinco espécies florestais usadas em projetos de reflorestamento, nos seus cinco compartimentos da biomassa: raízes, madeira, casca, galhos e folhagem. A análise de variância entre os teores de carbono das espécies e dos compartimentos mostrou variação entre $39 \mathrm{e}$ $49 \%$, e indicou diferenças significativas entre os teores das espécies e dos compartimentos. Ficou comprovado que precisam ser tratados de forma específica os teores por espécies e por compartimentos quando da realização das estimativas para projetos.
\end{abstract}

Palavras-chave: teor de carbono, compartimentos, espécies florestais.

\section{Carbon Contents of Five Forest Species and Their Compartments}

\begin{abstract}
Biomass and carbon quantification are important for assessing the potential of carbon sequestration for forest species. Part of biomass corresponds to the carbon that can be converted to $\mathrm{CO}_{2}$ equivalent for use in CDM projects and other carbon offsets. The purpose of this study was to evaluate the carbon content of five tree species used in reforestation projects in five biomass compartments: roots, wood, bark, branches, and foliage. Through the analysis of variance of the species, it was possible to notice that the carbon content ranged from 39 to $49 \%$, which indicates significant differences between the species and the carbon contents of the compartments. It was demonstrated that, in order to be treated, the contents of species and compartments need to be specified, when carrying out estimates for projects.
\end{abstract}

Keywords: carbon content, biomass compartments, forest species. 


\section{INTRODUÇÃO}

As florestas, de acordo com a definição proposta pelo acordo de Marraqueche (United..., 2001) e a definição da FAO (Food..., 2004), prestam um serviço de captura e fixação de carbono na madeira e nos demais componentes da biomassa, sendo que essa situação pode ser considerada como uma forma de mitigação das mudanças climáticas. A quantificação do carbono orgânico presente nas mesmas é importante, uma vez que, durante a permanência dessas florestas, existe uma grande quantidade de carbono fixado que deixa de estar presente na atmosfera (Caldeira et al., 2003). Por esse fato, torna-se importante o conhecimento sobre o potencial das florestas de armazenar carbono, uma vez que, a partir dessas informações, podem ser elaborados novos projetos de florestamento ou reflorestamento, visando à mitigação das mudanças climáticas (Weber et al., 2006) e à comercialização de créditos de carbono (Gorgens et al., 2005).

O carbono assimilado pode ser usado para crescimento e estabelecimento de reservas, sendo que a magnitude da taxa de assimilação líquida depende da constituição fisiomorfológica das espécies vegetais, tais como capacidade fotossintética, área foliar e proporção de tecidos fotossintetizantes na folhagem (Larcher, 2000). Além disso, fatores ambientais como água e nutrientes, por exemplo, podem afetar a produção de matéria seca e, consequentemente, interferir no balanço de carbono. Ainda acerca desses fatores, uma maior radiação luminosa (intensidade e/ou exposição) é capaz de promover aumentos no incremento de biomassa, já que espécies com elevadas taxas de crescimento relativo precisam de mais luz para alcançar os respectivos ótimos metabólicos (Taiz \& Zeiger, 2009).

Geralmente, as estimativas de carbono fixado na biomassa florestal podem ser obtidas por meio da multiplicação do valor da biomassa encontrado por um teor de carbono. De forma genérica, muitos trabalhos vêm adotando ou recomendando esse teor como um valor genérico de $50 \%$ do peso da biomassa (Gorgens et al., 2005, Fukuda et al., 2003; Soares \& Oliveira, 2002; Fang et al., 2001). Quando não se tem a informação da quantidade de carbono para a espécie de interesse, o Intergovernmental Pannel On Climate Change - IPCC (1996) recomenda o uso do fator 0,5 para converter biomassa seca em carbono. (Gorgens et al., 2005).

Entretanto, a adoção de um valor geral pode acarretar superestimativas ou subestimativas de estoques de carbono, ou seja, uma fonte de erro que pode ser evitada se os teores corretos forem empregados.

Rochadelli (2001), ao estudar a estrutura de fixação de carbono em reflorestamentos de Mimosa scabrella Benth (bracatinga), concluiu que diferentes classes sociais apresentam diferentes concentrações dos constituintes fundamentais e acidentais da biomassa tanto da madeira quanto da casca na espécie estudada, sendo que a concentração de carbono pode variar em torno de 40 a $45 \%$ da biomassa seca total.

A literatura demonstra que existem muitas variações em termos de estoques de biomassa, em diferentes plantios sujeitos a diferentes condições de desenvolvimento, como afirmado por Watzlawick et al. (2005) e Saidelles et al. (2009). Os teores de carbono, apesar de apresentarem algumas variações, são bem mais estáveis, oscilando entre 39 e $51 \%$, com média em torno de $45 \%$ da biomassa seca (Watzlawick et al., 2003). Em função da importância que a variável teor de carbono possui na estimativa dos estoques de carbono presentes na biomassa florestal e, em função das diferenças entre os teores de carbono encontrados em estudos científicos, torna-se importante que mais estudos demonstrem, para as mais diversas situações, esses valores.

Dessa maneira, o objetivo deste trabalho foi quantificar o carbono em cada compartimento das árvores, sendo: casca, folhagem, galhos vivos, madeira e raízes. Também foi objetivo deste trabalho avaliar os padrões de agrupamento dos teores de carbono das cinco espécies utilizadas em plantios florestais. As hipóteses testadas neste trabalho são de que existem diferenças significativas nos teores de carbono para as cinco espécies testadas, bem como nos teores de carbono dos cinco compartimentos das árvores. 


\section{MATERIAL E MÉTODOS}

O presente estudo foi desenvolvido com base em um conjunto de dados de teores de carbono de cinco espécies florestais com diferentes números de indivíduos por espécie e variações de idade e de povoamentos. Essas espécies são comumente usadas em reflorestamentos no sul do Brasil, sendo: 40 indivíduos da espécie Pinus taeda L. (Pinus) variando entre 2 e 24 anos; 30 indivíduos da espécie Mimosa scabrella Benth (Bracatinga) variando entre 2 e 19 anos; 45 indivíduos da espécie Populus deltoides W. Bartram ex Marshall (Álamo) variando entre 2 e 8 anos; 24 indivíduos de Eucalyptus grandis W. Hill (Eucalipto) variando entre 8 e 20 anos, e 10 indivíduos da espécie Araucaria angustifolia (Bertol.) Kuntze (Araucária) variando entre 14 e 31 anos.

Para realizar a determinação dos teores de carbono, foi necessário coletar previamente diversas amostras de biomassa das espécies em questão. Para a determinação da biomassa, foi utilizado o método direto, também chamado de destrutivo.

Depois de selecionados os indivíduos representativos de cada povoamento, realizou-se o abate do mesmo, sendo separados e pesados folhas, casca, madeira, galhos e raízes. Esses compartimentos da biomassa foram fracionados e encaminhados para laboratório para a determinação dos teores de carbono.

A amostragem de folhas foi feita coletando-se subamostras na ponta, no meio e na base dos galhos localizados no terço médio da copa. A amostragem dos galhos acompanhou o mesmo procedimento adotado para as folhas. As amostras da casca e da madeira do fuste foram retiradas de discos com $5 \mathrm{~cm}$ de espessura, retirados na base, à meia altura e na ponta de cada árvore. A biomassa radicial foi quantificada na área determinada como área útil de cada planta dentro do povoamento; no caso, com um plantio de $3 \times 2 \mathrm{~m}$, a área útil é de $6 \mathrm{~m}^{2}$, que foi escavada até $1 \mathrm{~m}$ de profundidade, onde as raízes com diâmetro igual ou superior a $1 \mathrm{~cm}$ foram coletadas.

Para determinação do teor de carbono total das amostras, foi utilizado um equipamento especializado para a análise de carbono, $\mathrm{o}$ analisador de carbono LECO, modelo C-144, o qual possui um software para registro digital dos resultados. O equipamento analisa o carbono pela combustão total, na qual a amostra é submetida à temperatura de $1.000{ }^{\circ} \mathrm{C}$ por cerca de 60 segundos. Nesse método, a amostra de material sólido é levada à combustão, sendo que um sensor detecta a quantidade de dióxido carbono $\left(\mathrm{CO}_{2}\right)$ gerado, relacionando automaticamente com a quantidade de carbono elementar existente na amostra.

Foram analisadas 753 amostras, para as cinco espécies em seus cinco compartimentos, conforme está apresentado na Tabela 1. As análises estatísticas descritivas e experimentais foram processadas por meio do programa Excel 2003 com o suplemento Action 1.0. Os testes de comparação de média foram realizados com o software Biostat 5.0, a fim de avaliar suas medidas de tendência central e dispersão, e verificar se, estatisticamente, existiam diferenças significativas entre os teores de carbono, entre os compartimentos de cada espécie e entre os teores médios de cada espécie.

Com base nas análises estatísticas, foi realizada a análise de agrupamento das espécies e por compartimento. Existem várias formas de agrupamento, sendo que uma das mais utilizadas é a distância euclidiana (Hair et al., 1998), a qual foi

Tabela 1. Quantidade de amostras realizadas por espécie e por compartimento.

Table 1. Number of samples taken by species.

\begin{tabular}{lccccccc}
\multicolumn{1}{c}{ Espécies } & Folhagem $^{\star}$ & Casca & Madeira & Galhos & Raízes & Total \\
\hline Araucaria angustifolia & 10 & 10 & 10 & 10 & 10 & 50 & 120 \\
\hline Eucalyptus grandis & 24 & 24 & 24 & 24 & 24 & 30 & 158 \\
Mimosa scabrella & 30 & 30 & 29 & $39^{* *}$ & 40 & 40 & 200 \\
\hline Pinus taeda & 40 & 40 & 40 & 45 & 45 & 225 \\
\hline Populus deltoides & 45 & 45 & 45 & 158 & 149 & 753 \\
\hline Total & 149 & 149 & 148 & 158 \\
\hline
\end{tabular}

${ }^{*}$ Acículas em coníferas e folhas para as folhosas. ${ }^{*}$ Foram agrupados os teores de carbono de galhos grossos e finos. 
utilizada neste estudo. A distância euclidiana é a raiz quadrada da soma dos quadrados das diferenças de valores para cada variável.

\section{RESULTADOS E DISCUSSÃO}

Na Figura 1, estão apresentados os teores médios de carbono para cada espécie e compartimento analisados. Constatou-se que os teores de carbono das espécies analisadas raramente ultrapassaram valores de $50 \%$, sendo comum encontrar valores entre 39 e 50\%. Para o gênero Populus sp., os maiores teores foram encontrados nos galhos, com média de 45,05\%. No caso da espécie Araucaria angustifolia, os maiores teores foram encontrados nas acículas (46,78\%). A espécie Mimosa scabrella foi a que apresentou as menores variações entre os teores de carbono e a que apresentou, em média, o maior teor de carbono (45\%).

A folhagem do gênero Eucalyptus grandis apresentou o maior teor encontrado, com cerca de $51,29 \%$. Para a espécie Pinus taeda, o valor mais alto encontrado para o carbono presente na biomassa foi de $49,56 \%$, presente no compartimento casca.
Higuchi \& Carvalho Junior (1994) analisaram diversas espécies da Floresta Tropical Úmida Densa de Terra Firme, na região de Manaus, e observaram um teor médio de carbono para o tronco e os galhos de $48 \%$, e, nas folhas, de $39 \%$.

Já Balbinot et al. (2003) encontraram, para Pinus taeda com 5 anos de idade, teores médios para acícula, galhos, casca, madeira e raízes de 47,3, 43, 40, 45,7 e 42,8\%, respectivamente.

Weber et al. (2003) analisaram os teores de carbono orgânico em povoamentos de Araucaria angustifolia (Bert.) Ktze, em diferentes idades, encontrando os teores médios para fuste, folhagem, galho morto, galho vivo, raiz e casca de: $44,1,44,0,42,9,41,7,40,5$ e 40,1\%, respectivamente.

Sette Junior et al. (2006), comparando estatisticamente diferentes idades de Pinus taeda com relação aos teores médios de carbono, encontraram teores bem elevados em relação a outros trabalhos com essa espécie, sendo encontrado um teor médio de $55,3 \%$. Uma das justificativas encontradas pelos autores para um teor elevado foram as condições de sítio, o material genético, o tipo e o manejo, que contribuíram para tais teores.

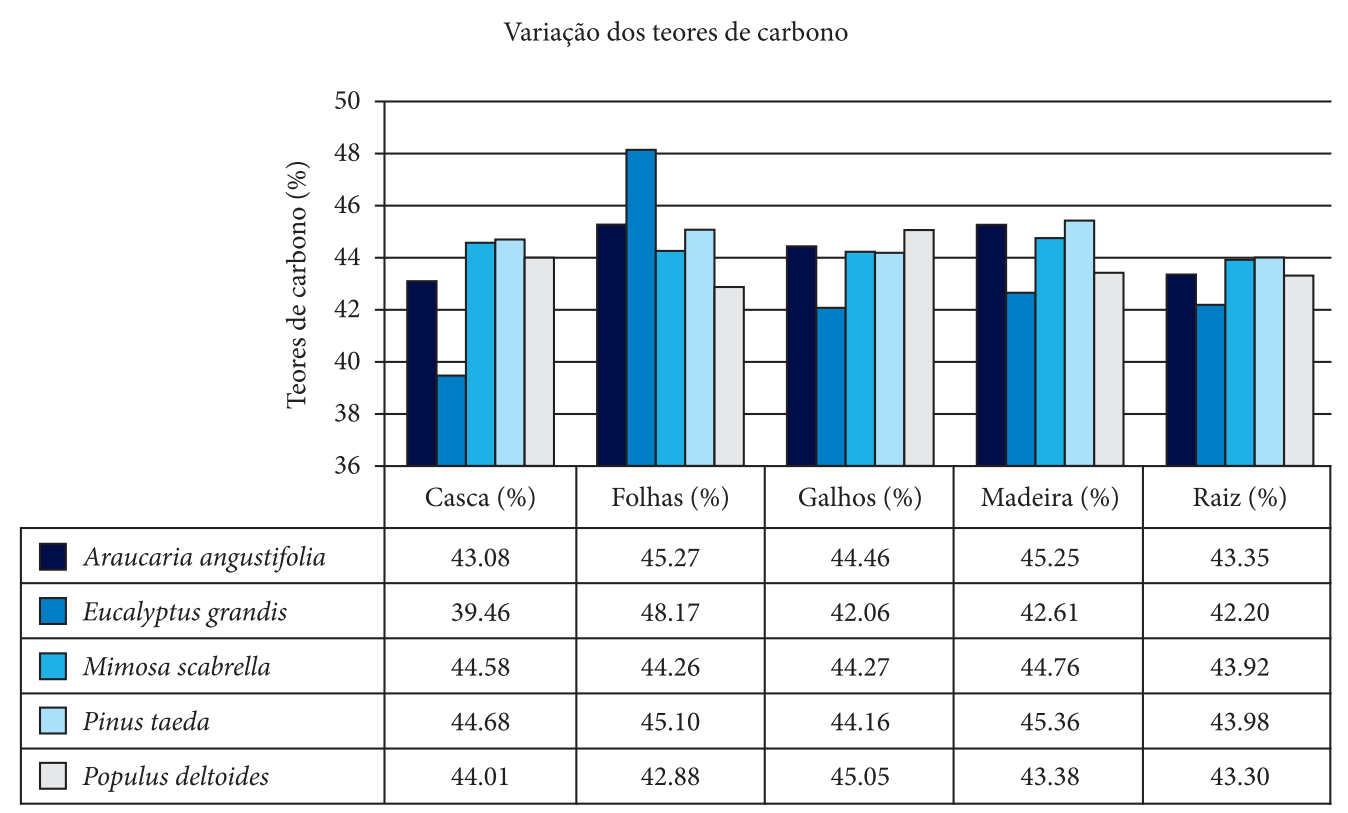

Figura 1. Valores médios encontrados para o teor de carbono por compartimento. Figure 1. Values found for carbon content per compartment. 
Weber et al. (2006), analisando seis espécies naturais do ecossistema da Floresta Ombrófila Mista, encontraram valores para casca, folha, fuste, galho morto, galho vivo e serrapilheira que, em termos gerais, apresentaram valores médios entre 39,9 e $42,2 \%$, sendo que nas seis espécies os teores de carbono no compartimento das folhas apresentaram valores entre 41,4 e $46,1 \%$.

Saidelles et al. (2009), estudando os teores de Acacia mearnsii De Wild., com 4 anos de idade, encontraram teores para os compartimentos folha, galho vivo, galho morto, casca, madeira e raiz de 45,8, $41,39,40,87,40,68,42,13$ e 41,92\%, respectivamente. Os resultados de Almeida et al. (2010) indicam valores de 32,7 e 44,7\% para a serapilheira de um povoamento de Tectona grandis L.F.

Os teores encontrados neste trabalho, comparativamente com os outros trabalhos citados, confirmam que dificilmente existem teores que ultrapassam valores de 50\%. De maneira geral, também foi possível observar que existe tendência em relação à alocação de carbono nos compartimentos da biomassa florestal, pois os teores apresentados no compartimento folhas são evidentemente superiores aos encontrados nos outros compartimentos. Essa observação de que as folhas ou acículas apresentaram os maiores teores de carbono em relação aos demais compartimentos foi também constatada por Taiz \& Zeiger (2009). A explicação desses autores para esse fato foi que uma vez que o processo fotossintético se desenvolve nesse compartimento, era de se esperar que os maiores teores estivessem presentes no mesmo. Conforme complementam, a rota de fixação normal entre os compartimentos seria: folhagem, galhos, madeira raiz e casca.

Uma das justificativas apontadas para as diferenças observadas é citada por Watzlawick et al. (2005), que afirmam que diferentes tipos de florestas armazenam quantidades diferentes de carbono; o mesmo conceito pode ser aplicado aos indivíduos, pois a porção da árvore em que o carbono é armazenado também varia, pois tal teor está relacionado com a quantidade de biomassa presente em cada compartimento. Essas informações se corroboram, pois os grupos de espécies respondem diferentemente às condições de sítio a que são submetidas (Selle, 2007).

\subsection{Análise de variância e teste de médias}

Para verificar e analisar as diferenças entre as espécies, foi realizado um teste de comparação de médias entre os compartimentos. A comparação evidenciou as diferenças existentes entre os teores de carbono para as espécies e para os compartimentos no nível de 99 e 95\% de probabilidade, sendo a significância de cada comparação dada pelo teste de Tukey.

Os resultados da ANOVA avaliaram todas as combinações, de forma que todos os compartimentos de cada uma das espécies foram comparados. Para o compartimento folha, apenas a comparação entre P. taeda e A. angustifolia, e entre $M$. scabrella e E. grandis não apresentou uma diferença significativa entre os teores de carbono. Para o compartimento casca, E. grandis foi a única espécie que apresentou uma diferença significativa no nível de $99 \%$ para os teores de carbono em relação às outras espécies. Para o compartimento madeira, não houve uma diferença significativa entre as espécies $P$. taeda, $P$. deltoides e M. Scabrella, e a comparação entre as outras espécies deste trabalho demonstra diferenças significativas nos níveis de 95 e 99\%. As diferenças também se encontram no compartimento galhos entre $P$. taeda, $P$. deltoides e E. grandi, e entre E. grandis, M. scabrella e A. angustifolia, observam-se diferenças significativas entre os teores de carbono. Para as raízes, foram encontradas diferenças significativas entre $P$. taeda e E. grandis, entre $M$. scabrella, $P$. deltoides, E. grandis e A. angustifolia, e entre $P$. deltoides e E grandis.

\subsection{Análise de agrupamento}

Após as analises estatísticas e as comparações feitas pelo teste de comparação de médias, foi possível evidenciar que existem diferenças entres os teores de carbono para as espécies avaliadas. Após a análise de agrupamentos realizada pelo método distância euclidiana (Hair et al., 1998), foi observada, de forma que foram feitas duas análises de agrupamento distintas: uma entre as espécies deste estudo e uma entre os compartimentos das espécies.

A existência da diferença estatística dos teores de carbono entre as espécies pode ser observada na Figura 2, na qual se percebe a distinção de três grupos, sendo estes: um grupo entre Pinus taeda 
e Araucaria angustifolia, outro grupo para Mimosa scabrella e Populus deltoides e outro grupo para Eucalyptus grandis. Observou-se que espécies de grupos semelhantes como, no caso, as coníferas, possuem um comportamento de fixação de carbono nos seus compartimentos (casca, folhagem, galhos madeira e raízes) diferente de espécies de folhosas.

Com relação aos compartimentos da biomassa (Figura 3), também houve a separação dos teores de carbono em três grupos distintos, de acordo com as diferenças estatísticas, sendo que o primeiro

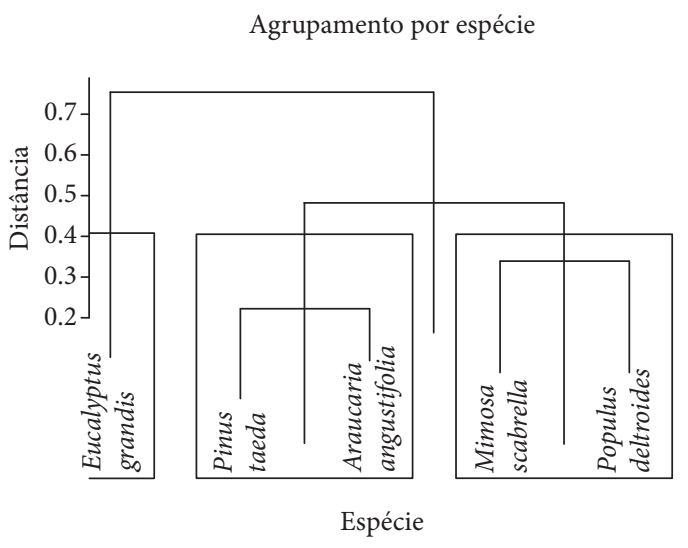

Figura 2. Agrupamento das espécies em função do teor de carbono.

Figure 2. Grouping of species for carbon content.

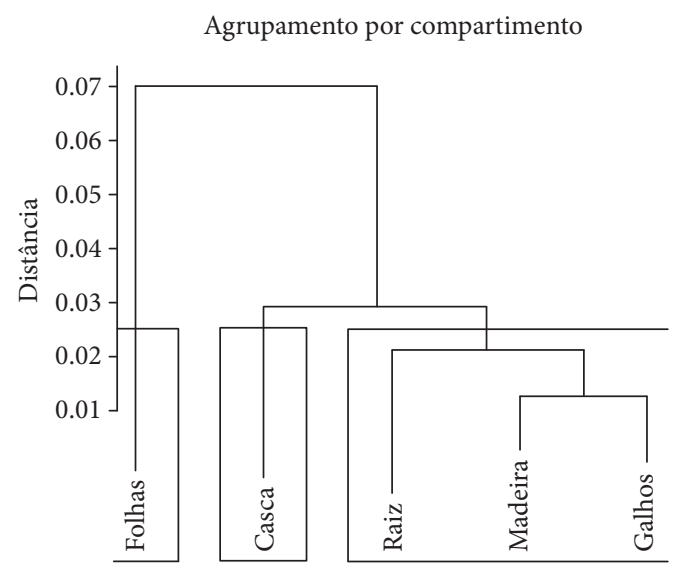

Compartimento

Figura 3. Agrupamento de compartimentos em função do teor de carbono.

Figure 3. Grouping of compartments for carbon content. grupo foi do compartimento folhagem, dado aos valores elevados em comparação com todos os compartimentos; o segundo foi do compartimento casca e o terceiro, dos compartimentos raízes, madeira e galhos.

Consequentemente, a distinção facilita o estudo e a compreensão da contribuição de cada componente da biomassa nas quantidades de carbono que são fixadas, bem como permite fazer estimativas mais acuradas sobre os estoques presentes para as espécies e para os seus compartimentos.

\section{CONCLUSÕES}

É possível observar que existem diferenças significativas entres os teores de carbono, por espécies e por compartimentos, apontando para a necessidade de usos de teores específicos quando são necessárias estimativas precisas sobre estoques de carbono.

A espécie que apresentou o teor mais elevado de carbono em toda a sua biomassa foi a Mimosa scabrella, cujos teores oscilaram entre 44,68 e $45,33 \%$, demonstrando que quando somente os teores forem levados em consideração, essa espécie apresenta alto potencial para fixação de carbono.

Os maiores teores de carbono foram observados nos compartimentos casca, madeira e raízes na espécie Pinus taeda, com 44,68, 45,36 e 43,98\%, respectivamente. Os menores teores médios foram observados na espécie Eucalyptus grandis, nos compartimentos casca, galhos, madeira e raízes, com $39,46,42,06,42,61$ e $42,20 \%$, respectivamente.

Quando da necessidade de utilização de um teor médio para as espécies avaliadas neste trabalho, conclui-se que pelo menos seja adotado um valor distinto entre coníferas e folhosas, em função do desempenho dos resultados nas análises de agrupamento.

\section{STATUS DA SUBMISSÃO}

Recebido: 29/11/2010

Aceito: $12 / 11 / 2011$

Resumo publicado online: 16/11/2011

Artigo completo publicado: 22/12/2011 


\section{AUTOR(ES) PARA CORRESPONDÊNCIA}

\section{Felipe Scheibe Dallagnol}

Programa de Pós-graduação em Ciência Florestal, Universidade Federal do Paraná - UFPR,

Av. Lothário Meissner, 632, Jardim Botânico,

CEP 80210-170,

Curitiba, PR, Brasil

e-mail: fsdallagnol@gmail.com

\section{REFERENNCIAS}

Almeida EM, Campelo JH, Finger Z. Determinação do estoque de carbono em teca (Tectonagrandis L. F.) em diferentes idades. Ciência Florestal 2010; 20(4):559-568.

Balbinot R, Schumacher MV, Watzlawick LF. Inventário do carbono orgânico em um plantio de Pinus taeda aos 5 anos de idade no Rio Grande do Sul. Ciências Exatas e Naturais 2003; 5(1):59-68.

Caldeira MVW, Schumacher MV, Barichello LR,Vogel HLM. Determinação de Carbono Orgânico em Povoamentos de Acaciamearnsii de Wild. plantados no Rio Grande do Sul. Revista Acadêmica: ciências agrárias e ambientais 2003; 1(2):47-54.

Fang J, Chen A, Peng C, Zhao SC. Changes in forest biomass carbon storage in China between 1949 and 1998. Science 2001; 292(5.525):2.320-2.322.

Food and Agriculture Organization-FAO. Global Forest Resources Assessment Update 2005, Terms and Definitions. Roma: FAO; 2004. Working Paper 83/E. [cited 2011 maio 01]. Available from: http://www.fao. org/forestry/7797-0f7ba44a281b061b9c964d3633d8 bf325.pdf.

Fukuda M, Iehara T, Matsumoto, M. Carbon stock estimates for sugi and hinoki forests in Japan. Forest Ecology and Management 2003; 184(1-3):1-16. http:// dx.doi.org/10.1016/S0378-1127(03)00146-4

Gorgens EB, Oliveira MLR, Leite HG, Brum Neto MS. Metodologia para Monitoramento do Estoque de Carbono: Revisão e Estudo de Caso. Biomassa \& Energia 2005; 2(2):169-175.

Hair J, Black W, BabinB, Anderson R, Tatham R. Multivariate Data Analysis. New Jersey: Prentice Hall; 1998.

Higuchi N, Carvalho Junior, JA. Fitomassa e Conteúdo de Carbono de Espécies Arbóreas da Amazônia. In: Anais do Seminário "Emissão x Sequestro de CO2 - Uma Nova Oportunidade de Negócios para o Brasil”, 1994; Rio de Janeiro. Rio de Janeiro; 1994. p. 127-153.
Intergovernmental Panel On Climate Change IPCC. 1996 guidelines for national GHG inventories: Reference Manual. <http://www.ipcc-nggip.iges.or.jp/ public/gl/invs6.htm>. [Acesso em: 01 ago. 2011].

Larcher W. Ecofisiologia vegetal. São Carlos: Rima Artes e textos, 2000.

Rochadelli R. A estrutura de fixação dos átomos de carbono em reflorestamentos: Estudo de caso: Mimosa scabrella Benth, bracatinga [tese]. Curitiba: Universidade Federal do Paraná; 2001.

Saidelles FLF, Caldeira MVW, Schumacher MV, Balbinot R. Uso de equações para estimar carbono orgânico em plantações de Acaciamearnsii De Wild. no Rio Grande do Sul - Brasil. Revista Árvore 2009; 33(5):907-915. http://dx.doi.org/10.1590/S0100-67622009000500013

Selle GL. Ciclagem de nutrientes em ecossistemas florestais. Bioscience Journal 2007; 23. [cited 2011 maio 11]. Available from: http://www.seer.ufu.br/index. $\mathrm{php/biosciencejournal/article/view/6912/4577.}$

Sette Junior C, Nakajima N, Geromini M. Captura de carbono orgânico em povoamentos de Pinus taeda L. Na Região De Rio Negrinho, SC. Revista Floresta 2006; 36. [cited 2011 maio 11]. Available from: http://ojs.c3sl. ufpr.br/ojs2/index.php/floresta/article/view/5506/4036.

Soares CPB, Oliveira MLR. Equações para estimar a quantidade de carbono na parte aérea de árvores de eucalipto em Viçosa, Minas Gerais. Revista Árvore 2002; 26(5):533-539.

Taiz L, Zeiger E. Plant Physiology. Sinauer Associates, Inc, Publishers; 2009.

United Nations Framework Convention on Climate Change - UNFCCC. The Marrakesh Accords e The Marrakesh Declaration, 2001. [cited 2011 maio 01]. Available from: http://unfccc.int/cop7/documents/ accords_draft.pdf.

Watzlawick LF, Sanquetta CR, Arce JE. Carbono orgânico em povoamentos de Araucaria angustifolia (Bertol.) O. Kuntze no sul do Estado do Paraná, Brasil. Revista Acadêmica: ciências agrárias e ambientais 2003; 1(2):63-68.

Watzlawick LF, Sanquetta CR, Caldeira MVW. Estoque de carbono orgânico e biomassa em Pinus taeda L. Biomassa e Energia 2005; 2(1):7-17.

Weber KS, Balbinot R, Watzlawick LF, Sanquetta CR. Teores de carbono orgânico de seis espécies naturais do ecossistema da Floresta Ombrófila Mista. Ambiência 2006; 2(2):167-177.

Weber KS, Sanquetta CR, Mello AA, Watzlawick LF, Balbinot R. Variação nos teores de carbono orgânico em povoamentos de Araucaria angustifólia (Bert.) O. Ktze. Brasil Florestal 2003; (76):23-28. 